\title{
The Realization of Industrial Design Product with Computer Software
}

\author{
Jiangning $\mathrm{Li}^{1}$, Jingsong Huang ${ }^{2}$ \\ ${ }^{1,2}$ Hubei University of Technology, Hubei Wuhan, 430068
}

KEYWORDS: Industrial Design Product, Computer Software, Realization

\begin{abstract}
With the computer software and hardware technology development and CAD technology is widely used in industrial design, industrial design ways and means to achieve constantly updated development, industrial design field is undergoing a new revolution. Introduction: With the rapid development of the computer, so that industrial design computerized reached a considerable level. Computer data analysis, model building, into the production system, etc., in an important part of human life and production, resulting in a more wide-ranging impact, and thus lead to new thinking is gradually penetrate the industrial design subject areas.
\end{abstract}

\section{Introduction}

Computer aided product design refers to computer software and hardware-based, designer in the design process, with a new modern design approach computer involved in the development of new products, which in order to improve efficiency and enhance scientific and design reliability, adapt to the information society for the purpose of production. Computer expression in product design, the main products tend to form, color, material and other design elements of the simulation, in today's society plays a leading role in the design of earth manner.

With the participation of progress and designers of computer technology, computer design has become the most important change in signs, in terms of design concepts, or in the design methods and procedures are designed to bring a new philosophy, a comprehensive impact on all aspects of the design field. Of course, as the high-tech low-intelligence computer, design thinking in terms of expression has some limitations in the design only as a "secondary" designer tool is applied.

The traditional design approach is through the two-dimensional expression, and then made into a solid model, then improved results-based model, and then made into a drawing for the production, so that the process of making a two-dimensional model to express them, human error is considerable, when drawing engineering drawings designers consider optimization needs to make the right judgment by a detailed calculation and analysis skills, because sometimes often difficult to retreat. The computer-aided design of the intervention, so that we achieve a truly three-dimensional design, and any details of the product in front of the computer can be exhaustively demonstrated in front of the designer, and can be adjusted at any angle and position, shape, color, you can make timely changes in terms of texture, proportion, scale and so on. In the pre-production design drawings, a computer can be established for a three-dimensional model that you optimized structure design, greatly reduce design time and effort and more accuracy. And some professional design software, such as UG, Pro/E, can directly mold design and manufacture, so that design efficiency greatly improved. 


\section{Connotation and Characteristics of Industrial Design Products}

In the next period of time, the industrial design is the use of multi-disciplinary comprehensive technology, the pattern is: product-centric to the quality of the designer's assurance to the product data management and integration for the characteristics of virtual reality technology the main means of testing and performance analysis, three-dimensional computer model with CAM data exchange system designed for start and end, from design and development to achieve integrated production, integration and networking. This is the current general industrial design approach is fundamentally different.

This process can be seen from the design and development process of a product to go through frequent changes in personnel and premises and the like. The new product design and development process and the above process are fundamentally different, the process of market research, put forward by the buyer (user) requirements for product design, "the designer product model and system builders in the computer" using virtual reality technology to simulate verify product performance and strength, stiffness, reliability, optimization and other technical requirements of tests and corrective feedback to design and communicate with the buyer at any time through the network technology, computer-aided process planning (CAI) "computer simulation machine" computer simulation delivery model for computer-aided manufacturing system, producing products.

As can be seen from the above process features of the new design are: ${ }^{1}$ designer products are three-dimensional computer model with CAM data exchange system, rather than drawings or computer graphics plane, data products in digital form in computer systems transmission, paperless design; new product designs is a comprehensive application of CAD, CAE, CAPP, CAM and other technology, product design must be a large computer integrated manufacturing system (CIMs) design of virtual reality (VR) technology is the design an important means; the product design process for the user is that the buyer can supervise, propose modifications to the process involved in setting juice; product design process people and places relatively stable, greatly shorten the design cycle; due to the application of VR technology, can easily and at low cost a lot of experiments, increase product reliability; $\dot{c}$ Since the user fully involved in the design process, before the product is not produced its performance has been very understanding, so as to use the product and for training period may be shortened; À certain product designer or design team will be relatively fixed; Á design group Head of experience and knowledge play a decisive role in the design of the key, and grasp the whole process of design.

\section{Development Trend of Design Technology}

In fact, now the field of design, especially in some large product design, it has been more or less reflect the above characteristics. The most typical example is the development of the Boeing 777 aircraft. It is in accordance with aircraft components product development team, a large number of applications CAD / CAM technology, simulation technology and virtual manufacturing technology, aircraft overall and 3 million parts, from design to processing the full realization of free drawing. Established through the use of a virtual three-dimensional solid model is really a variety of aircraft performance were analyzed, simulation, shortening the thousands of hours of work and save a lot of money.

\section{Effect of Computer Software for the Realization of Industrial Design Product}

The biggest impact of this new technology revolution may be the buyer of all the products will be designed as the center. As already mentioned, not only the starting point for a new design approach 
is to propose requirements for products by the buyer, and the buyer can participate in and supervise the whole process of design, with the use of new technology is relatively mature, a lot of preliminary design and easy to use software and virtual appear real products, so buyers can easily put forward higher requirements more reasonable, and timely design make the appropriate changes. In addition, before the product is not produced, the buyer has been considerable understanding of product performance and features, help the buyer to develop appropriate design commercialization strategies. Thus, the buyer has become the true masters of the beginning until the final use of the product design.

Future product development cycles, fast and convenient design process, so that the new product has faster speed, accelerate the inevitable product updates, users have more choice. In addition, the use of virtual reality technology matures, but also to simplify the training process, the training period may be shortened, the user performance requirements of the product will continue to improve, promote competition.

Due to a variety of convenient universal design software and improve the appearance of some of the primary design artifacts become as easy as stacking wood, in which the condensation degree of labor complexity will be reduced, resulting in social engineering and technical personnel of the general design work decline in value, most likely a lot of work in the design of "blue-collar technology."

Mentioned in the general design engineer downward social mobility does not mean that the overall decline in the value of industrial design. On the contrary, due to the design of more sophisticated and improved to increase the technical content of the design integrated into the overall value of the industrial design but increased. Therefore, the design and the core staff responsible for not only have deep technical expertise and rich experience in the design, but also require knowledge and ability to organize and coordinate interdisciplinary and orientation, will play a leading role in the design and development, determines the design the direction of development. Product development, especially the development of large products, required by the industry or product development specially designed systems, and personnel needed to design specialized training, such inputs is huge. Therefore, it is reasonable to infer that the future is bound to focus on product design, most large master in accordance with its range of products in a large enterprise or a small number of colleges and universities combined fixed design group, the formation of a new "technological monopoly."

\section{The Realization Conditions of Industrial Design Product}

Professional design software soils have pro / E, UG, Solid Works, Alias, etc. Pro/E is Pro / Engineer Abbreviation, developed by the United States PTC's only a core set of mechanical design automation software products, which in parametric and feature-based modeling technology to provide a revolutionary approach to designers to achieve mechanical design automation. it consists of a series of products consisting of modules, specifically applied to products from design to manufacturing process. The only data structure Pro / E provides the integration of all projects, thereby making the entire product closely together from design to manufacturing. Pro / E can always generate a 3D model into 2D drawing, automatic dimensioning, because of its associated properties, and the use of a single database, modify any dimensions, drawings, assembly drawings will change accordingly.

Alias Design Studio (Alias) is a very professional industrial design animation and simulation software, developed by the Canadian company Alias, the software must be early to run on high-performance computers, but because of the performance of the personal computer gradually 
improve, Alias8 .5 NT version officially released, so that Alias software application entered another new era. Alias industrial design, animation, sculpture, interior design, architectural design and other fields, has been dominant in the engineering design, it is good at expressing conceptual design phase, the designer can quickly sketch idea to a realistic three-dimensional model presented in front of.

UG is introduced by Unigraphics Solutions as a set of CAD / CAEICAM as one of the three-dimensional mechanical design platform, is the world's most advanced computer-aided design, analysis and manufacture of software, widely used in aerospace, automotive, shipbuilding and other fields. UG is an interactive computer-aided design (CAD), computer aided engineering (CAE) and computer-aided manufacturing (CAM) systems. It has most of the engineering design and drafting capabilities required in today's field of mechanical processing .UG is a full three-dimensional, double-precision manufacturing system, allowing users to compare accurately describe any geometric shape, molded by a combination of these and you can product design, analysis and mapping. UG can provide mechanical design, mold design and electrical design complete set of gun design, analysis, manufacturing solutions: UG offers a variety of modeling methods including feature-based modeling, surface modeling, solid modeling, including, while providing top-to lower and bottom-up assembly design methods, but also provides a powerful support for rendering, materials, textures, animation, background, visualization parameter settings for the product design renderings output.

Solid works is a well-known three-dimensional CAD software development vendors Solid works company posted market-leading 3D mechanical design software, Solid works is based on the Windows platform, the full parametric feature modeling software, it is very easy to achieve complex three-dimensional parts solid modeling, complex assemblies and Creating a drawing. The software can be applied to the rules of geometry-based mechanical product design and production preparation work. Solid works release the creativity of designers and engineers, so that they only spend a fraction of the time required for similar software to design better, more attractive, more popular in the market of the product.

\section{Conclusion}

Will appear any new technology is an opportunity for a new direction. Is a challenge, especially for a multi-disciplinary and cross technology is in its infancy. For future design, the biggest challenge may not be funds and technology, but the human factor. To implement the new revolutionary industrial design, human requirements are many, especially in terms of human consciousness. People accustomed to the idea or thought in the present situation may be behind the development of technology, which can be seen in every new technology appears in history. It is also unable to give a clearer realization of the time the most important factor.

\section{References}

[1] Listen Huang. Network interface cable color design study [D] Kunming: Kunming University, 2005.4.

[2] Song Ling, interactive web design visual experience [D] Xi'an: Xi'an Academy of Fine Arts, 2010.3.

[3] $\mathrm{Li} \mathrm{Li}$. Art materials and the material and its application in the study of art and design in the [D] Changchun: Jilin University, 2006.6.

[4] Wu Xiaoyan. Modern modeling and simulation technology [M]. Northwestern Poly technical University Press Society .2001. 